\title{
The Future of Cognitive Therapy and Research is Bright and Clear
}

\author{
Stefan G. Hofmann
}

Published online: 4 July 2012

(C) Springer Science+Business Media, LLC 2012

Founded in 1977, Cognitive Therapy and Research has become an established, respected, high-quality interdisciplinary journal that focuses on the investigation of all aspects of mental disorders and their treatments with a family of interventions known as behavioral and cognitive therapy (CT). This treatment approach, pioneered by Dr. Aaron T. Beck who serves on our Board of Associate Editors, has become the most effective therapeutic approach for virtually all mental disorders.

CT is one of the greatest success stories in psychology in part because it is firmly grounded in science. In fact, it could be argued that CT has evolved throughout the years from a treatment approach to a mature scientific discipline. Carefully controlled experimental studies using cuttingedge technologies to examine cognitive processes have become standard tools to many of my colleagues, as are randomized controlled trials examining the efficacy of specific CT techniques. The willingness to embrace the scientific method and to open ourselves up to scientific scrutiny by formulating testable theories has contributed to the success of CT.

However, despite this clear success, there is still room for further improvement and many important questions still remain unanswered. For example, combining CT and traditional pharmacotherapy is typically not much more effective, and can be even detrimental in the long-term, as compared to monotherapy. A better understanding of the treatment mechanisms can shed light on this and might further enhance the efficacy of CT. The search for the

S. G. Hofmann $(\bowtie)$

Department of Psychology, Boston University,

Boston, MA, USA

e-mail: shofmann@bu.edu generative mechanism is not limited to psychological constructs, but also includes physiological, biological, and genetic variables. In addition, contextual, cultural, and social factors need to be considered when investigating mental disorders.

These factors are closely linked to a fundamental shift that has occurred in psychology. This shift emphasizes research on emotions, emotion regulation strategies (such as acceptance, reappraisal, suppression, mindfulness, etc.) and the interplay between emotions, cognitions, behaviors, and social factors. In fact, psychology might be undergoing an emotional revolution, and neuroscience has begun to merge with psychology and emotion research. The formulation of the new field of affective neuroscience, which examines the biological correlates of affective states and emotions, is certainly a telling example. I am convinced that Cognitive Therapy and Research will soon become a major outlet for studies that reflect these new trends that are driven by research on emotion regulation, affective neuroscience, neurobiological correlates of $\mathrm{CT}$, and neuromarkers of CT response.

I am very excited about the new challenges and yet humbled and honored for having the privilege to serve as editor of Cognitive Therapy and Research. Thanks to Dr. Ingram and his excellent editorial team, the journal is in excellent shape. We receive approximately 200 unsolicited manuscripts per year, and the journal has a solid impact rating and strong visibility. My stellar editorial team and I invite submissions from diverse areas of psychology focusing on cognitions, cognitive processes, and behavioral and cognitive therapies. In addition, I strongly encourage submissions focusing on treatment mechanisms and predictors, experimental psychopathology, biomarkers, neuroscience, genetics, and pharmacotherapy. In addition, the scope of the journal includes dissemination, as well as 
developmental and cultural aspects of psychopathology and treatment. If you have ideas for special issues on cutting-edge research topics, please let me know; a number of them are already in the works.
The future of CT is indeed bright and clear. On behalf of my editorial team, we are looking forward to receiving your high-quality submissions. Cognitive Therapy and Research is the ideal outlet for your research. 